\title{
The overtraining reversal effect in the pigeon'
}

\author{
D. I. WILLIAMS \\ THE UNIVERSITY OF HULL, ENGLAND
}

The overtraining reversal effect was demonstrated with the pigeon, using a color discrimination. The effect was dependent upon the particular non-correction procedure used, which allowed responding in the intertrial interval when stimuli were absent. This resulted in rapid elimination of position habits arising on reversal.

Schade \& Bitterman (1965) demonstrated the overtraining reversal effect (ORE) in the pigeon using a shape discrimination, but found reversal on a color problem was retarded by overtraining. They used a modified correction or "guidance" procedure in which, after each incorrect response in the two-key situation, the positive stimulus was presented alone and a response to it reinforced. This procedure therefore ensures sampling of the positive stimulus, which may be particularly important after reversal, when incorrect responding to the old positive stimulus usually predominates. As Gossette \& Cohen (1966) pointed out, this procedure should facilitate reversal; they demonstrated that pigeons showed successive error reduction over a series of reversals on a spatial discrimination if a non-correction procedure were used. The present experiment investigates whether the ORE with a visual discrimination can be demonstrated in the pigeon without the use of a correction procedure.

\section{Method}

Ss (experimentally naive hybrid pigeons maintained at $85 \%$ of their free-feeding body weight) were trained in individual wire mesh cages measuring $2 \times 2 \times 2 \mathrm{ft}$. A hardboard sheet was fitted in place of the normal door; this covered the front of the cage completely. It had two circular apertures $1 \mathrm{in}$. in diameter, with their centers $4 \mathrm{in}$. apart and 6-1/2 in. above the floor of the cage. Between these and 2 in. below them was an aluminum feeding cup $1 \times 1 \times 1-1 / 2$ in. which was supplied from a hopper attached to the back of the board. The stimuli were drawn onto white paper which was fixed behind a clear Perspex strip. This could be moved in runners behind the board so as to expose the stimuli at the aperture. Each stimulus consisted of four circles, $1 / 4 \mathrm{in}$. in diameter, drawn in lines $1 / 32$ in. wide, arranged in a square with their centers $3 / 8$ in. apart. The two circles on the left (to the bird) were black. For one stimulus (B) the other two were blue, and for the other (R) one was red and one blue, red being at the top. The pigeon responded directly at the stimulus, receiving a single maple pea for each correct response. All operations of the apparatus were manual.

Each bird was fed only in the daily experimental session, and was given as many trials as it would perform in this time ( average 120). Pretraining was by means of a seed lure in the stimulus aperture; position habits were not eliminated at this stage. A non-correction training procedure was used, the stimuli being withdrawn between trials to leave a plain white ground. The intertrial interval was defined as 3 sec. after the last peck at the aperture; this had the effect of extinguishing responses to the "empty" aperture and largely eliminating position habits. Training was divided for computing purposes into blocks of 10 reinforced trials and criterion was defined as one block with one or no errors.

Six birds (criterion group) were reversed on reaching criterion, and six (overtrained group) received a further 500 reinforced trials before reversal. Half the birds in each group were trained initially with stimulus (R), and half with stimulus (B) positive.

\section{Results}

The results are shown schematically in Fig. 1. There was no significant difference in trials to criterion on the initial problem between the two groups (Mann-Whitney $U$ test, $U=17, n_{1}=n_{2}=6, p=.938$, twotailed test). The overtrained group learned the reversed



Fig. 1. Mean responses to criterion on initial problem (shaded column) and on reversal (open column). 
discrimination in fewer trials than the criterion group $(\mathrm{U}=4, \mathrm{p}=.013$, one-tailed test). There was a difference in the ratio of positive to negative responses between the groups; the overtrained group made more errors than correct responses in reaching criterion after reversal $(U=8, p=.06$, one-tailed test), whereas this was not so for the criterion group $(U=15, p$ $=.35$, one-tailed test).

\section{Discussion}

The ORE was demonstrated using what was basically a color discrimination. The assumption made by Schade \& Bitterman (1965) that the pigeon has such a relatively strong set for color that this dimension is not influenced by overtraining is therefore untenable. The finding that negative responses predominate in the overtrained group on reversal confirms the analysis of overtraining and extinction made by Mackintosh (1963).

There are a number of possible reasons why the procedure used here should have resulted in an ORE. It might have been due to the degree of overtraining: 500 trials, as against 200 in the Schade \& Bitterman study. Alternatively, it might be a function of stimulus complexity, for it follows from the Sutherland-Mackintosh theory (Mackintosh \& Mackintosh, 1963) that a complex situation is necessary for the ORE to occur. However, the writer (1966) has used exactly the same experimental design, except that a correction procedure was used with no interval between trials, and failed to show an ORE. The critical feature would therefore appear to be the use of this particular noncorrection procedure; moreover, the way the intertrial interval was defined seems especially important. As a result of training, birds very seldom responded to the stimulus aperture when the stimulus was absent. However, soon after the problem was reversed, they reverted to responding in the intertrial interval. Welldefined position habits could be identified in this period. It would seem, then, that as a result of reversal, birds revert to position strategies. In the overtrained group these tend to be very persistent, which might be expected if it was argued that reversal produces greater frustration in the overtrained group. The method used here, of extending the intertrial interval until no responses are made for a given time, quickly extinguishes these position habits. Without this procedure, any facilitation of reversal resulting from overtraining might be offset by the development of these strong position habits.

\section{References}

Gossette, R. L., \& Cohen, H. Error reduction by pigeons on a spatial successive reversal task under conditions of non-correction. Psychol. Rcp., 1966, 18, 367-370.

Mackintosh, N. J. Extinction of a discrimination habit as a function of overtraining. J. comp. physiol. Psychol., 1963, 56 , 842-847.

Mackintosh, N. J., \& Mackintosh, J. Reversal learning in octopus vulgaris with and without irrelevant cues. Quart. J. cxp. Psychol., 1963, 15, 236-242.

Schade, A. F., \& Bitterman, M. E. The relative difficulty of reversal and dimensional shifting as a function of overlearning. Psychon. Sci., 1965, 3, 283-284.

Williams, D. I. Some aspects of stimulus analysis in pigeons. Unpublished doctoral thesis. University of Hull. (1966)

\section{Nofe}

1. This work was carried out under a D. S. I. R. studentship. I would like to express my thanks to my supervisor, Mr. W. A. Matthews. 